\title{
Thermogénèse et obésité : mécanismes moléculaires
}

Le tissu adipeux brun est un organe spécialisé dans la production de chaleur chez les nouveau-nés. L'utilisation de techniques de biologie moléculaire permet d'envisager une approche nouvelle des relations entre le dysfonctionnement des adipocytes bruns et l'établissement de l'obésité.

\section{Daniel Ricquier}

Maître de conférences à l'université Pierre-et-Marie-Curie

\section{REFEERENCES}

I. Sims EAH. Experimental obesity, dietary induced thermogenesis and their clinical implications. Clin Endocrinol Metab 1976; 5 : 377-96.

2. Garrow JS. The regulation of energy expenditure in man. In: Bray GA, ed. Recent advances in obesity research. Westport: Food and Nutrition Press, 1978 : 200-10.

3. James WPT. Clinics in Endocrinology and Metabolism, Vol. 13, Number 3. London : Saunders, 1984 .

\section{ADRESSE}

Université Pierre-et-Marie-Curie, laboratoire de physiologie comparée (CNRS-U.A. 307). 4, place Jussieu. 75230 Paris Cedex 05.

$\mathrm{m} / \mathrm{s}$ mai 85 'obésité est un problème de santé majeur dans le monde occidental. Le risque de mortalité est accru chez les obèses à cause de la plus grande fréquence de troubles tels l'athérosclérose coronarienne, l'hypertension et le diabète de type II. Malgré son importance pour la santé de la population, les facteurs essentiels responsables du développement de l'obésité ne sont pas encore tous bien connus. Conceptuellement, le problème est simple : l'obésité est la conséquence d'une situation où l'apport d'énergie à l'organisme a excédé la production d'énergie pendant une période de temps assez longue. S'il est patent que dans certains cas d'obésité, le surpoids est bien dû à une suralimentation, il est tout aussi clair que dans de nombreux cas, un tel comportement alimentaire ne peut expliquer l'obésité. En effet, il a été montré que des individus avec des prises alimentaires très inégales, peuvent contrôler leur poids corporel. Inversement, avec des apports alimentaires égaux et à activité physique égale, certaines personnes régulent leur poids, d'autres grossissent. De telles observations ont conduit à penser que la capacité d'un individu à contrôler son poids est liée à la facilité qu'il a à moduler sa dépense énergétique [I-3]. La dépense énergétique a trois composants : le métabolisme de base, l'activité physique et la thermogénèse. Le métabolisme de base est un peu plus élevé chez les individus obèses. L'activité physique n'est généralement pas différente chez les individus sédentaires obèses ou normaux. Ainsi, ni des variations du métabolisme de base, ni des variations de l'activité physique, ne peuvent expliquer un éventuel défaut de la dépense énergétique chez l'obèse. Par contre, des travaux récents permettent de poser l'hypothèse que certains obèses présentent un défaut de dissipation d'énergie sous forme de chaleur. Un tel trouble contribuerait alors à l'accumulation de lipides.

Le but de cet article est de rappeler brièvement les relations existant entre la thermogénèse et l'obésité et, d'autre part, d'expliquer un exemple de mécanisme moléculaire impliqué dans ces relations.

\section{Thermogénèse sans frisson}

La thermogénèse dépend principalement de la température ambiante et de l'apport alimentaire. L'abaissement de la température externe induit chez les homéothermes une production de chaleur destinée à maintenir constante la température centrale. Celle-ci comprend d'une part, la chaleur produite par le frisson thermique musculaire et, d'autre part, la thermogénèse sans frisson ou thermogénèse chimique dont les effecteurs ne sont pas parfaitement identifiés.

La prise d'aliments est un des fac- 


\section{RÉFÉRENCES}

4. Girardier L, Stock MJ. Mammalian Thermogenesis. London : Chapman and Hall, 1983.

5. Rothwell N, Stock MJ. A role for brown adipose tissue in the diet-induced thermogenesis. Nature 1979; 281 : 31-5.

6. Landsberg L, Young JB. The role of the sympathoadrenal system in modulating energy expenditure. Clin Endocrinol Metab 1984; $13: 475-99$.

7. Jéquier E. Energy expenditure in obesity. Clin Endocrinal Metab 1984; $13: 563-80$.

8. Felig P. Insulin is the mediator of feedingrelated thermogenesis : insulin resistance and/or deficiency results in a thermogenic defect which contributes to the pathogenesis of obesity. Clin Physiol 1984; $4: 267-73$.

9. Danforth EJr, Burger A. The role of thyroid hormones in the control of energy expenditure. Clin Endocrinol Metab 1984; 13 : 581-95.

I0. Himms-Hagen J. Brown adipose tissue thermogenesis, energy balance and obesity. $\operatorname{Can} 7$ Biochem Mol Biol 1984; 62 :610-7.

II. Trayhurn P. The development of obesity in animals : the role of genetic susceptibility. Clin Endocrinol Metab 1984; $13: 451-74$.

12. Jung RT, Shetty PS, James WPT. Reduced thermogenesis in obesity. Nature 1979; 279 : 322-33.

13. Lindberg O. Bromn Adipose Tissue. New York : Elsevier, 1970.

14. Nicholls DG, Locke $R$. Thermogenic mechanisms in brown fat. Physiol rev 1984; $64: 1-64$.

15. Ricquier D. The brown adipose tissue mitochondrial uncoupling protein. In: Trayhurn P, Nicholls DG, eds. Brown Adipose Tissue. London teurs majeurs qui stimule la thermogénèse chez les mammifères. Après un repas, la production de chaleur s'accroît. Ce phénomène est désigné sous le nom de thermogénèse induite par l'alimentation (en anglais, Diet-induced thermogenesis ou DIT). Les autres expressions qui ont été utilisées pour ce même processus sont l'action dynamique spécifique (qui contrairement à ce qu'on pensait n'est pas spécifique des protéines), la thermogénèse postprandiale et l'effet thermique de l'alimentation. La thermogénèse induite par l'alimentation comprend une composante "obligatoire" et une composante "facultative". La première est la dépense énergétique due à la digestion, absorption et transformation métabolique des aliments. La seconde, encore appelée thermogénèse variable, est la fraction de la thermogénèse induite par la prise de nourriture qui ne peut être comptabilisée dans la fraction " obligatoire ". Cette thermogénèse facultative est liée à la quantité et aux qualités gustatives des aliments. Elle permet à l'organisme de dissiper sous forme de chaleur l'énergie apportée par un excès d'alimentation. Ce phénomène sert donc directement à limiter le gain de poids corporel lors d'un apport calorique excessif. Au début du siècle, Neumann avait postulé l'existence d'un tel mécanisme physiologique, alors nommé luxuskonsumption.

La thermogénèse sans frisson induite par le froid et la thermogénèse induite par l'alimentation, présentent plusieurs points communs. Dans les deux cas, des centres nerveux hypothalamiques et les nerfs orthosympathiques interviennent. Il est bien établi que le système sympathique est essentiel à la production de chaleur chez l'animal exposé au froid [4] et chez l'animal faisant de la thermogénèse induite par les aliments [4, 5]. Par ailleurs, chez les rongeurs, pour ces deux sortes de thermogénèse, l'organe effecteur principal est le tissu adipeux brun. La situation est moins claire chez l'homme, puisque l'existence des deux types de thermogénèse dans l'espèce humaine est controversée. Cependant, l'existence de la thermogénèse induite parol'alimentation chez l'homme a été récem- ment établie [2, 7]. Des travaux récents montrent, bien que cela soit encore discuté, l'existence d'un contrôle orthosympathique de cette thermogénèse [7]. Il a également été proposé que l'insuline [8] ou la $\mathrm{T}_{3}$ [9] sont des médiateurs de la thermogénèse induite par l'alimentation. Cependant, on ne connaît pas chez l'homme, les organes effecteurs de ce type de thermogénèse.

\section{Thermogénèse} et obésité

Pour démontrer l'existence d'un défaut de thermogénèse chez les mammifères obèses, les chercheurs disposent de nombreux modèles animaux correspondant à des rongeurs avec obésité expérimentale (obtenue par traitement neurochirurgical, chimique, endocrinien ou nutritionnel), ou bien avec obésité génétique. La plupart des rongeurs obèses présentent à la fois des défauts de thermogénèse sans frisson et de thermogénèse induite par l'alimentation [IO, I I]. Ainsi, les rats nourris avec un régime riche, varié et appétissant (régime cafétéria), peuvent développer une très forte thermogénèse et prendre peu de poids par rapport à l'apport calorique [5].

Il est donc admis que l'obésité de certains rongeurs est due en partie à une diminution de leur capacité à faire de la chaleur induite par l'alimentation. Ils ont alors une forte efficacité alimentaire conduisant à l'installation de l'obésité.

Pour l'espèce humaine, la situation est évidemment plus confuse. Certains auteurs n'ont pas décelé de diminution de la réponse thermogénique à l'alimentation chez des patients obèses. Par contre, d'autres auteurs tels Jéquier et coll. [7], ont observé que la réponse thermogénique au glucose et à l'alimentation était abaissée chez des obèses. Une telle perturbation. existe surtout chez des obèses chez qui la maladie est apparue pendant l'enfance. Le travail de Jéquier et coll. mérite une attention particulière, car il est basé sur l'utilisation de techniques de calorimétrie et de respirométrie modernes et précises. Par ailleurs, la thermogénèse induite par l'injection de noradrénaline est nettement 

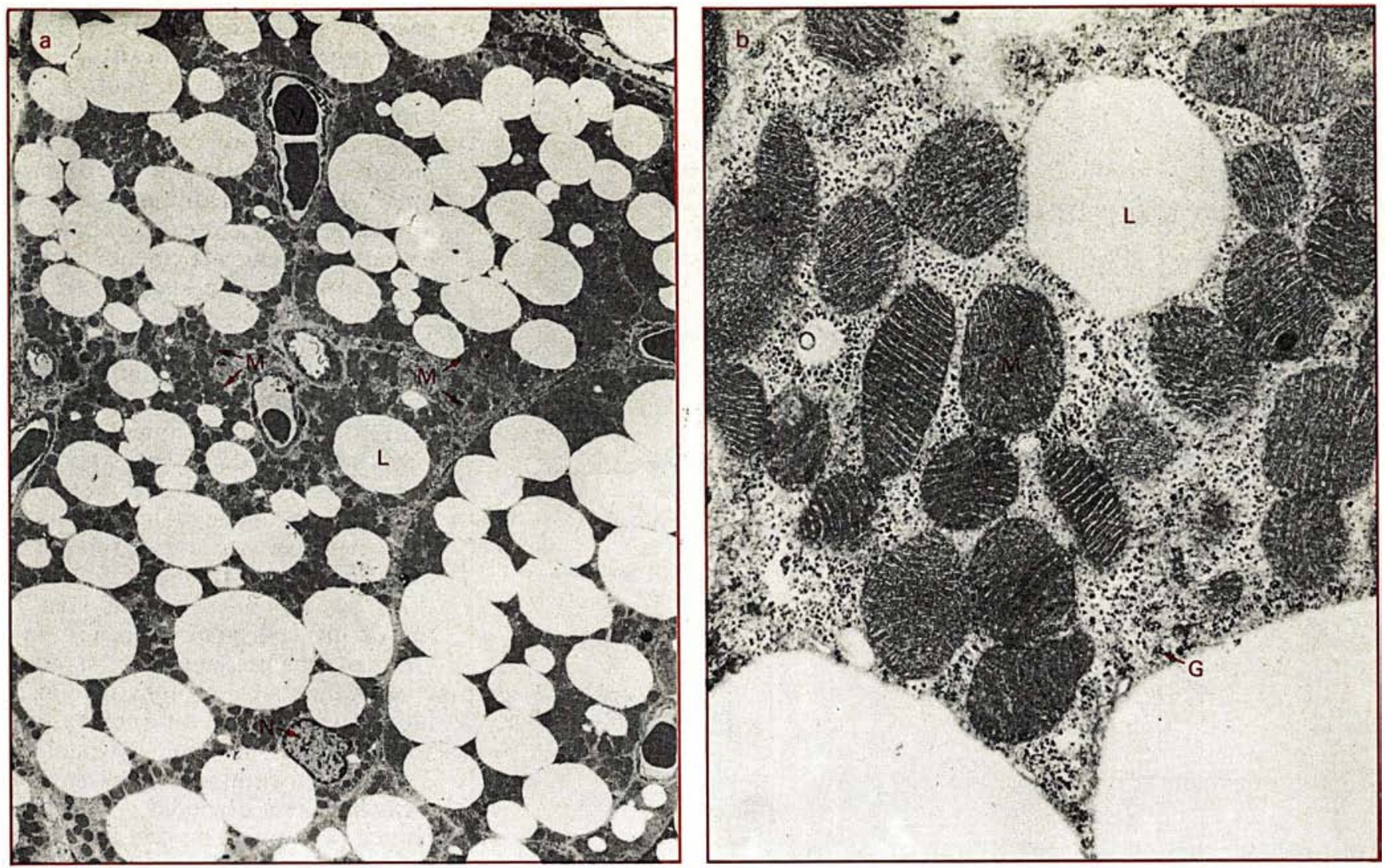

Figure 1. Micrographies électroniques de tissu adipeux brun interscapulaire de rat adapté au froid. A gauche (a) : $\times 2000$. A droite (b) : $\times 30000$.

$L=$ gouttelettes de lipides; $N=$ noyau; $M=$ mitochondries; $G=$ glycogène; $V=$ vaisseau capillaire.

Clichés du Dr M. Néchad. Figure extraite de Ricquier D, Mory G [2I].

réduite chez des femmes obèses ou ayant été obèses [12]. Ces observations conduisent des auteurs à proposer, comme cela a été fait pour les animaux obèses, qu'un défaut de thermogénèse peut contribuer au développement de l'obésité chez certains sujets prédisposés.

Si cette hypothèse est valable, il faut déterminer les mécanismes cellulaires et moléculaires dont le dysfonctionnement provoque une diminution de la thermogénèse. Récemment, deux choses ont été élucidées : d'une part le mécanisme moléculaire de production de chaleur par les adipocytes bruns, d'autre part le rôle du tissu adipeux brun dans la thermogénèse induite par l'alimentation chez les rongeurs. C'est pourquoi ces points, ainsi que l'intérêt de leur étude pour comprendre l'obésité humaine, vont être analysés maintenant.

Le tissu adipeux brun (TAB) est un $\mathrm{m} / \mathrm{s}$ mai 85 organe spécialisé dans la dissipation d'énergie sous forme de chaleur. Il est présent chez la plupart des mammiferes nouveau-nés (y compris l'enfant), chez les animaux hibernants et chez beaucoup d'animaux adultes adaptés au froid. Il a été clairement établi, entre I 962 et I970, qu'il est une source de chaleur importante, à la naissance, lors du réveil de l'hibernant et lors de l'exposition prolongée au froid. Le TAB peut représenter 0,5 à $5 \%$ du poids corporel. Il se trouve en quantité importante dans les régions interscapulaire, cervicale, thoracique, périaortique et périrénale. Le tissu est richement vascularisé et innervé. Des fibres orthosympathiques innervent les artérioles et les adipocytes bruns. Les adipocytes bruns (figure 1) sont caractérisés par la présence de gouttelettes de triglycérides et de mitochondries très nombreuses [13].
On sait que la production de chaleur est due à un découpage physiologique des phosphorylations oxydatives dans les mitochondries de l'adipocyte brun.

\section{Production de chaleur}

Dans les mitcchondries, à l'exception du TAB, le couplage entre l'oxydation des substrats et la phosphorylation de l'ADP, est dû à l'existence d'un gradient électrochimique de protons $\left(\Delta \mu_{H}+\right)$ de part et d'autre de la membrane mitochondriale (théorie chimioosmotique de Peter Mitchell). Suite à l'oxydation des substrats, des protons sont expulsés dans l'espace intermembranaire. Ces protons ne peuvent repasser librement dans la matrice. Ils ne peuvent rentrer que par la partie membranaire de l'ATP-synthase. A la suite de ce 


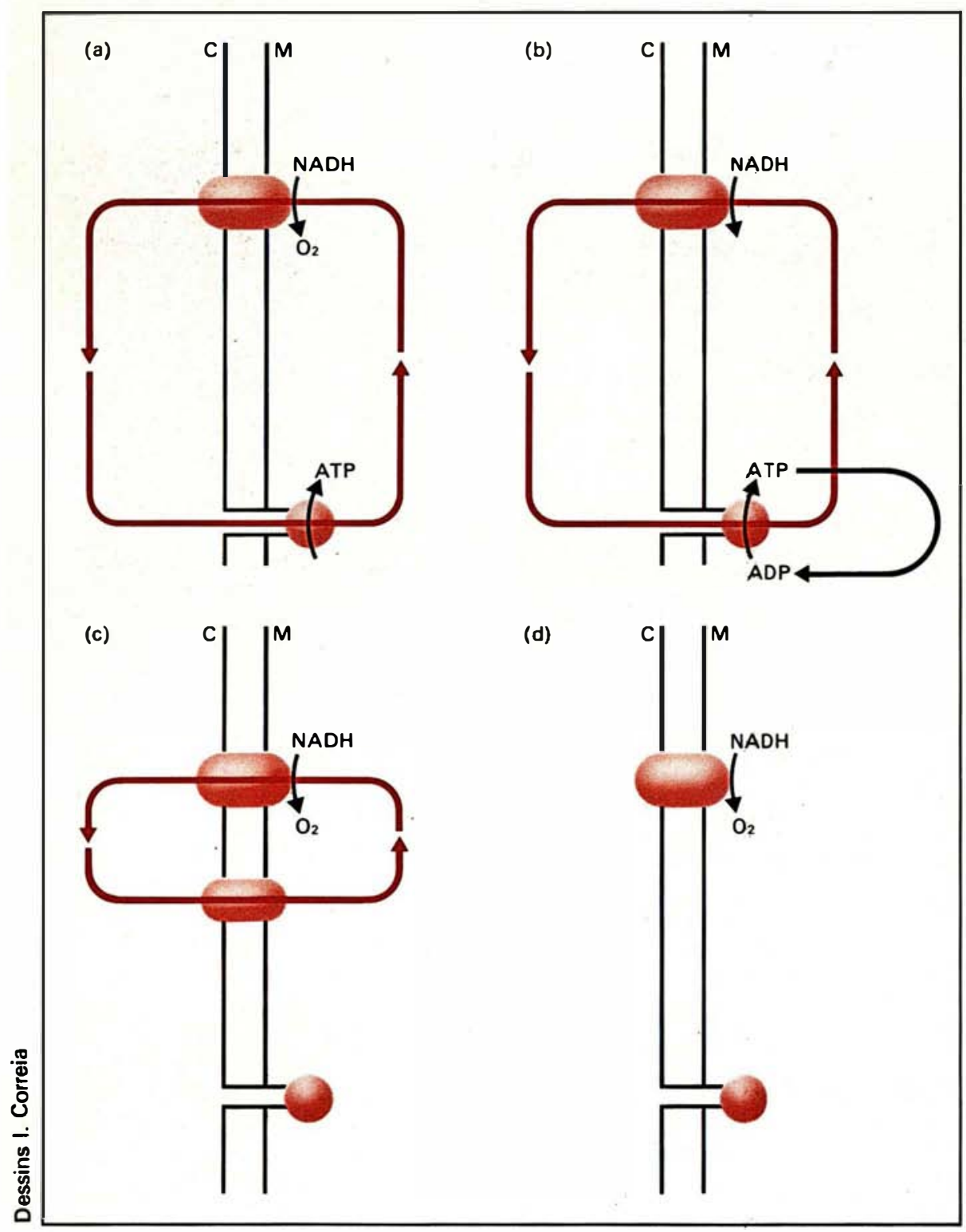

Figure 2. Circuits de protons à travers la membrane interne mitochondriale.

(a) : dans une mitochondrie normale, le couplage entre loxydation des substrats et la synthèse d'ATP est contrôlé par un circuit de protons.

(b) : respiration accélérée par une hydrolyse rapide de l'ATP.

(c) : absence de contrôle respiratoire due à lexistence d'une voie alternative (courtcircuit) de rentrée des protons dans la matrice mitochondriale.

(d) : absence de contrôle respiratoire due à une chaîne respiratoire modifiée n'expulsant pas de protons.

Les schémas (b), (c) et (d) indiquent différents moyens pour la mitochondrie de faire de la chaleur. Dans le cas des mitochondries de tissu adipeux brun, c'est le processus (c) qui est responsable de la dissipation d'énergie sous forme de chaleur. $C,=$ région cytoplasmique. $M=$ matrice mitochondriale. passage, l'ATP est généré. A l'état d'équilibre, la valeur de $\Delta \mu_{H}+$ est telle qu'elle induit la rentrée des protons à travers l'ATP-synthase (figure 2). David Nicholls [14] a montré que la perméabilité aux protons de la membrane interne mitochondriale des adipocytes bruns est anormalement élevée. Cela entraîne l'effondrement de $\Delta \mu_{H}+$ et le découplage. Ainsi cès mitochondries sont exceptionnellement capables de respirer très vite sans être obligées de synthétiser de l'ATP stæchiométriquement. La variation d'enthalpie correspondant à l'oxydation des substrats est alors dissipée sous forme de chaleur [14]. En fait, tout se passe comme si la membrane interne des mitochondries des adipocytes bruns était le lieu d'un court-circuit à protons. On sait maintenant que la forte conductance aux protons responsable du découplage, est due à la présence d'une protéine particulière dans la membrane interne des mitochondries du TAB. Cette protéine a pour poids moléculaire apparent 32000 , elle forme des dimères dans la membrane et est appelée protéine découplante. La protéine découplante est spécifique des mitochondries du TAB; elle a été purifiée et possède une forte capacité de liaison de certains nucléotides tels le GDP [I4, I5]. On peut résumer ainsi le processus d'activation de l'adipocyte brun conduisant au dégagement de chaleur: un ordre d'origine hypothalamique entraîne la libération de noradrénaline sur la membrane des adipocytes bruns. Après la fixation du neuromédiateur sur des récepteurs de type $\beta_{1}$, de l'AMP cyclique est produit, la lipase-hormone sensible est activée, et des acides gras libres sont formés à partir des triglycérides présents dans la cellule. Ces acides gras vont à la fois être les substrats des oxydations mitochondriales et les activateurs des canaux à protons par la protéine découplante [14]. L'augmentation de la conductance aux protons induit alors le découplage mitochondrial et la dissipation d'énergie sous forme de chaleur (figure 3).

Le TAB est le seul organe dont la fonction essentielle est la production de chaleur. Le rôle du TAB dans la 
thermogénèse sans frisson est parfaitement démontré, et les travaux récents de Foster établissent que la contribution du TAB à la production de chaleur induite par le froid est très grande [4]. Par ailleurs, la démonstration d'un rôle important de ce tissu dans la thermogénèse induite par l'alimentation a été faite par Rothwell et Stock en I979 [5].

\section{L'obésité des rongeurs et des hommes}

Ces auteurs ont montré que l'alimentation de type "cafétéria " provoque chez le rat une forte thermogénèse. Cette thermogénèse permet à l'animal de peu grossir. L'organe thermogénique impliqué dans une partie ou la totalité du phénomène est le TAB. Parallèlement, de nombreux travaux ont été effectués chez les rongeurs obèses. Tous ces travaux démontrent que le TAB des rongeurs obèses est très peu thermogénique [I0, I I, I 6]. Ainsi, chez les rongeurs, le TAB est un effecteur essentiel de la thermogénèse sans frisson, mais aussi de la thermogénèse induite par l'alimentation. Une diminution de l'activité thermogénique du TAB pourrait donc bien expliquer, au moins en partie, l'obésité des rongeurs.

L'organe de la thermogénèse induite par l'alimentation, chez les rongeurs, est donc le TAB. Si un défaut de ce mode de thermogénèse peut contribuer à l'établissement de l'obésité humaine, il faut identifier l'organe effecteur responsable. Ce problème n'a pas encore de solution, mais à titre d'hypothèse, un rôle du TAB chez l'homme peut être proposé.

Il est établi que le nouveau-né humain et le jeune enfant possèdent du TAB fonctionnel contenant la protéine découplante [17]. Cependant, deux questions se posent : l'adolescent et l'adulte humain possèdent-ils du TAB? Par rapport à des individus sains, l'adolescent et l'adulte obèses ont-ils un TAB peu développé ou défectueux? Nous ne connaissons pas la réponse à cette seconde question. En ce qui concerne la première question, différents travaux indiquent la présence d'adipocytes bruns chez de jeunes adultes $[18,19]$, ou bien chez

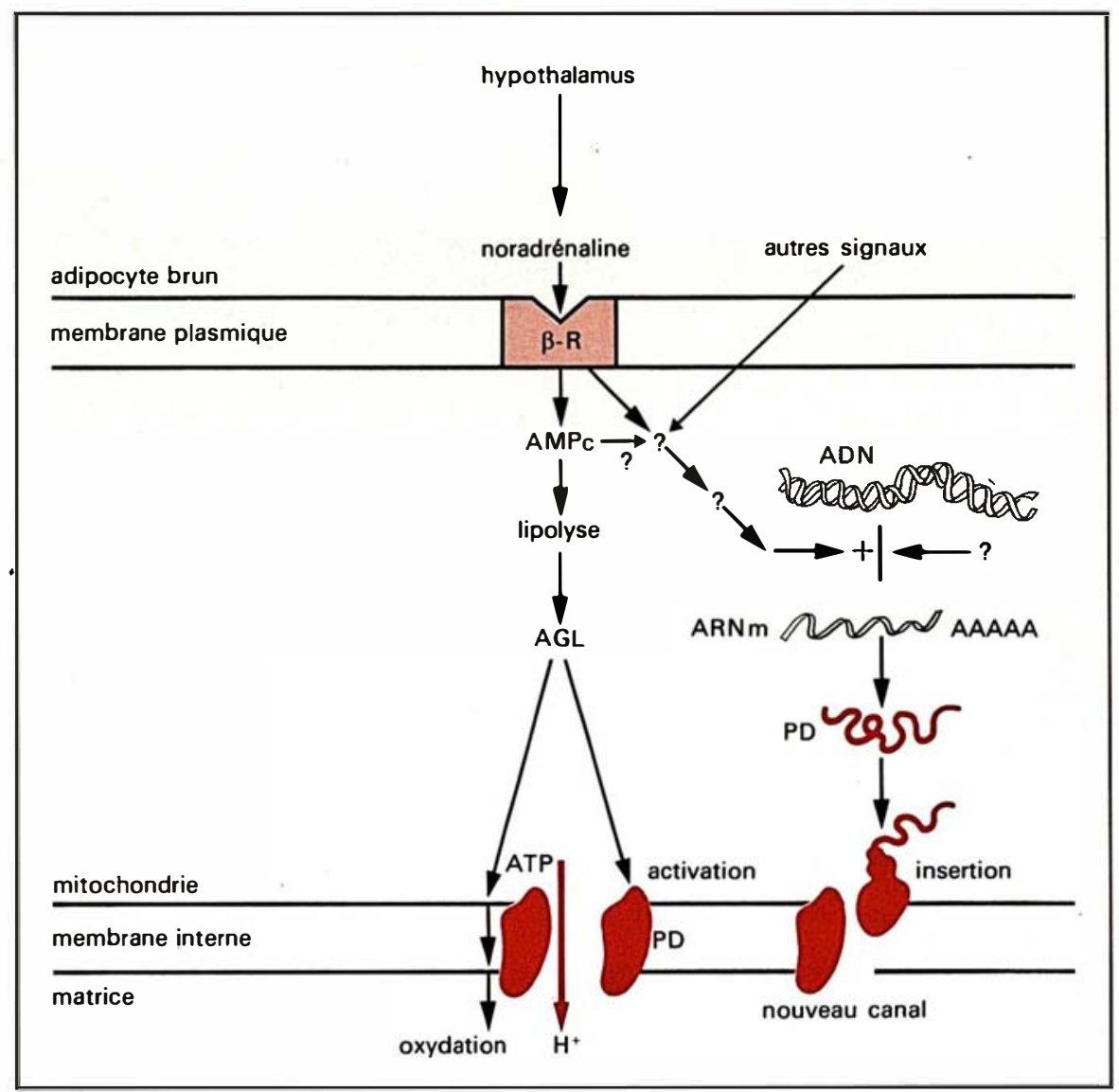

Figure 3. Mécanisme d'activation de l'adipocyte brun et de stimulation de la synthèse de la protéine découplante. Suite à la réception de signaux concernant la situation énergétique de lorganisme, de la noradrénaline est libérée à la surface des adipocytes bruns. Suivant la fixation du neuromédiateur sur les $\beta$-récepteurs $(\beta-R)$, la lipolyse est fortement activée. Les acides gras libres ( $A G L)$ produits sont à la fois les substrats des oxydations et les activateurs des canaux à protons formés par la protéine découplante (PD).

La rentrée des protons induit le découplage des phosphorylations oxydatives et la thermogénèse. La fixation de la noradrénaline sur les $\beta$-récepteurs stimule parallèlement la synthèse de l'ARN messager codant pour la protéine découplante.

des travailleurs d'extérieur du Nord ficative à l'équilibre ou au déséde la Finlande [20], ou encore chez quilibre énergétique de l'organisme. des patients adultes atteints de la La capacité d'un mammifere à faire maladie d'Addison ou porteurs de de la chaleur et à lutter ainsi contre phéochromocytomes [2I]. Toute- l'obésité pouvant être liée à la préfois, il est difficile, dans ces condi- sence de TAB, il est tout à fait tions, de calculer la capacité important de connaître les facteurs thermogénique totale et réelle du responsables du développement du TAB. On peut cependant souligner tissu et de la synthèse de la protéine

que la puissance du TAB activé découplante.

chez les rongeurs a été estimée à 400 watts par kilogramme de tissu [4], que le métabolisme de base d'un adulte humain de 70 kilogrammes correspond à 85 watts pour le corps entier, et donc qu'il suffirait que l'organisme humain renferme 50 à 200 grammes de TAB pour que celui-ci contribue de manière signi-

\section{Contrôle neuro-hormonal}

Des travaux effectués en bonne partie dans notre laboratoire démontrent que le développement du TAB est essentiellement contrôlé par l'innervation orthosympathique du 


\section{RÉFÉRENCES}

I6. Girardier L, Seydoux J. Is there a sympathetic regulation of the efficiency of energy utilization? Diabetologia $1981 ; 20: 362-5$.

17. Lean MEJ, James WPT. Uncoupling protein in human brown adipose tissue mitochondria. Isolation and detection by specific antiserum. FEBS Lett 1983; $163: 235-40$.

18. Heaton JM. The distribution of brown adipose tissue in the human. 7 Anat 1972; 112 : 35-9.

19. Tanuma Y, Ohata M, Ito T, Yokochi C. Possible function of human brown adipose tissue as suggested by observation on perirenal brown fats from necropsy cases of variable age groups. Archiv Histol Japon 1976; 39 : 1 17-45.

20. Huttunen $P$, Hirvonen J, Kinnula V. The occurrence of brown adipose tissue in outdoor workers. Eur 7 Appl Physiol 1981; 46 : 339-46.

21. Ricquier D, Mory G. Factors affecting brown adipose tissue activity in animals and man. Clin Endocrinol Metab 1984; $13: 501-20$.

22. Ricquier D, Néchad M, Mory G. Ultrastructural and biochemical characterization of human brown adipose tissue in pheochromocytoma. f Clin Endocrinol Metab 1982; 54 : 803-7.

23. Mory G, Bouillaud F, Combes-George M Ricquier D. Noradrenaline controls the concentration of the uncoupling protein in brown adipose tissue. FEBS Lett 1984; $166: 393-6$.

24. Young $P$, Wilson S, Arch JRS. Prolonged $\beta$-adrenoreceptor stimulation increases the amount of GDP-binding protein in brown adipose tissue mitochondria. Life $S_{c i}$ 1984; 34 : I I I I -7.

25. Ricquier D, Thibault J, Bouillaud F, Kuster Y. Molecular approach to thermogenesis in brown adipose tissue. Cell-free translation of mRNA and characterization of the uncoupling protein. 7 Biol Chem 1983; 258 : 6675-7.

26. Freeman KB, Chien SM, Litchfield D, Patel HV. Synthesis in vitro of rat brown adipose tissue $32000 \mathrm{M}_{\mathrm{r}}$ protein. FEBS Lett 1983; 158 : 325-30.

27. Bouillaud F, Ricquier D, Mory G, Thibault J. Increased level of mRNA for the uncoupling protein in brown adipose tissue of rats during thermogenesis induced by cold-exposure or norepinephrine infusion. $7 \mathrm{Biol}$ Chem 1984; 259 : 11 583-6.

28. Bouillaud F, Ricquier D, Thibault J, Weissenbach J. Molecular approach to thermogenesis in brown adipose tissue: cDNA cloning of the mitochondrial uncoupling protein. Proc Natl Acad Sci USA 1985; 82 : 445-8.
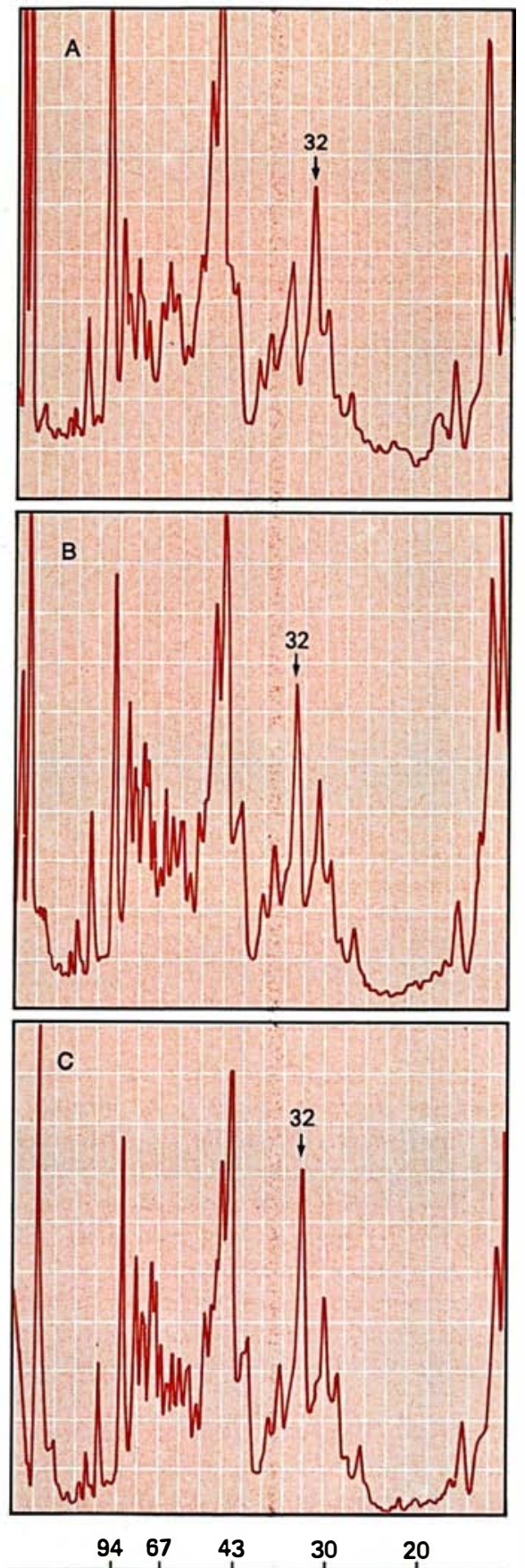

Figure 4. Augmentation du taux de protéine découplante chez le rat traité chroniquement par de la noradrénaline. Densitométrie des électrophorèses de protéines mitochondriales de tissu adipeux brun de rat témoin $(A)$, traité avec la noradrénaline $(B)$, exposé au froid $(C)$. La flèche 32 correspond à la protéine découplante de poids moléculaire apparent 32000.

Figure extraite de Mory G., [23]. tissu [2 I]. Lorsque des rats sont adaptés au froid, il y a formation de $\mathrm{TAB}$, i. e. multiplication de préadipocytes, différenciation en adipocytes bruns, synthèse de mitochondries et de protéine découplante. L'apparition de ces phénomènes est inhibée chez l'animal exposé au froid dont le TAB a été sympathectomisé. Par contre, ces phénomènes sont induits chez le rat non exposé au froid, mais porteur d'un phéochromocytome. Par ailleurs, reprenant les observations anciennes de Feyrter, nous avons démontré que le tissu adipeux périrénal de patients porteurs de phéochromocytomes est du TAB. Dans ce cas, les mitochondries des adipocytes bruns contiennent la protéine découplante [21, 22]. Tout récemment, il a été observé que la perfusion de rats avec de la noradrénaline induit le développement du TAB et la synthèse de la protéine découplante [23] (figure 4). Par ailleurs, une corrélation entre la capacité thermogénique du TAB et le taux de renouvellement de la noradrénaline dans le tissu a été démontrée [6]. Tout cela amène à conclure que la noradrénaline libérée par les termisaisons orthosympathiques à la surface des adipocytes et des préadipocytes bruns n'est pas seulement l'activateur métabolique de la cellule, mais est aussi l'inducteur du développement du TAB et de la synthèse de la protéine découplante [15, 2I] (figure 3). Cette conclusion est confirmée par l'observation d'un développement de la capacité thermogénique du tissu chez des rats traités avec un nouveau $\beta$-agoniste ayant une forte affinité pour les adipocytes bruns [24].

\section{Régulation moléculaire}

La compréhension des mécanismes de production de chaleur et de différenciation des adipocytes bruns a orienté une partie des recherches vers des aspects plus moléculaires. Ainsi l'utilisation des techniques de synthèse acellulaire et d'immunoprécipitation a permis de démontrer que, contrairement à ce qui est connu pour la plupart des protéines mitochondriales, la protéine découplante n'est pas synthétisée sous 


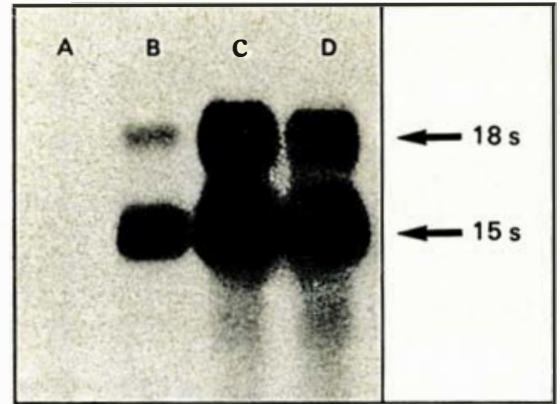

Figure 5. Hybridation d'une sonde d'ADNc aux ARN messagers de la protéine découplante de tissu adipeux brun de rongeur.

Des $A R N m$ extraits de foie (trace $A$ ) ou de tissu adipeux brun (traces $B, C$ et D) ont été électrophorésés dans un gel d'agarose en présence de formaldéhyde et transferrés sur de la nitrocellulose. Une séquence d'ADNc clonée et radioactive correspondant à l'ARNm de la protéine découplante a été ajoutée. Le filtre de nitrocellulose est ensuite autoradiographié. La sonde ne détecte aucun $A R N$ messager parmi les $A R N$ de foie $(A)$. Pour le tissu adipeux brun, 2 ARN messagers (I8 $S$ et $15 S)$ sont hybridés avec la sonde. On peut voir que le niveau de messagers codant pour la protéine est fortement accru dans le tissu hautement thermogénique ( $B$, animal témoin; $C$, animal exposé au froid et $D$, animal portant un phéochromocytome).

Figure extraite de Bouillaud [28].

forme de précurseur de plus haut poids moléculaire [15, 25-27].

Récemment, à partir d'ARN messagers de TAB fractionnés sur gradient de saccharose, des ADN complémentaires ont été préparés. Ensuite, ces $\mathrm{ADNc}$ ont été insérés dans des plasmides d'E. coli. Les clones bactériens contenant l'ADNc correspondant à l'ARNm de la protéine ont été isolés [28]. Nous disposons ainsi de sondes moléculaires spécifiques du messager de la protéine découplante. Ces sondes présentent plusieurs intérêts : d'une part elles permettent d'obtenir la séquence de la protéine, d'autre part elles permettent d'envisager l'étude du gène correspondant. Par ailleurs $\mathrm{m} / \mathrm{s} \mathrm{mai} 85$ les sondes d'ADNc obtenues donnent la possibilité d'identifier et de doser l'ARN messager de la protéine dans différentes situations physiologiques, pharmacologiques ou pathologiques. Un exemple est donné dans la figure 5. L'hybridation de la sonde radioactive à des ARN messagers indique que la transcription du messager de la protéine découplante est fortement stimulée chez des rats exposés au froid ou recevant des catécholamines. L'intérêt de ce genre d'approche est de fournir un outil d'étude des mécanismes d'activation et de transcription du gène de la protéine découplante et aussi d'envisager l'identification des adipocytes bruns fonctionnels chez l'homme.

\section{Conclusion}

Il a été montré ici comment un défaut de capacité thermogénique de l'organisme peut faciliter l'établissement de l'obésité chez l'animal et chez l'homme. Les études faites chez l'animal impliquent un défaut de production de chaleur induite par l'alimentation au niveau du TAB dans un tel processus. Pour l'espèce humaine, il reste à démontrer que le TAB joue un rôle important dans la thermogénèse induite par l'alimentation et dans la régulation du poids corporel. Les résultats obtenus au niveau du TAB des rongeurs sont cependant précieux pour essayer de répondre à cette question. En effet, les méthodes biochimiques, immunologiques et moléculaires mises au point pour identifier et doser la protéine découplante vont être utilisables chez l'homme. La connaissance du mécanisme de transcription du gène de la protéine découplante pourrait éventuellement déboucher sur un traitement pharmacologique induisant une augmentation de la capacité thermogénique des adipocytes bruns ou de cellules autres. Peut-être la reconsidération de l'obêsité en tant que déséquilibre énergétique, et les progrès dans la connaissance du TAB, vont-ils un jour conduire à une nouvelle attitude clinique devant le problème de certaines obésités

NDLA : Je remercie le.Docteur Gérard Mory d'avoir relu le manuscrit.

\section{Summary}

Certain kinds of obesity, in animals and in man, could be the result of a diminished capacity of the organism to dissipate excess food energy in the form of heat. In obese rodents, the defective thermogenic organ is brown adipose tissue. However, the role of brown adipose tissue in regulating body temperature in man has not been established. Brown adipose tissue is an organ specialized in the production of heat in newborns (including human newborns), in animals adapted to cold climates, and in hibernating animals. The production of heat is the result of a natural uncoupling of oxidative phosphorylations in the brown adipocytes. This uncoupling is due to the presence of a particular mitochondrial membrane protein: the uncoupling protein. The activation of brown adipocytes, the development of tissue and the synthesis of the uncoupling protein are controlled by norepinephrine. DNA sequences complementary to the messenger RNA of the uncoupling protein have been cloned in $E$. coli. These molecular probes have allowed scientists to study how the synthesis of the uncoupling protein is regulated, and also to evaluate the thermogenic capacity of the cells. The knowledge of the biology of brown adipose tissue and the use of the techniques of molecular biology should facilitate the studies of brown adipose tissue in the human adult and of the possible relationship between a dysfunction of these cells and the onset of obesity.

\section{TIRES A PART}

D. Ricquier : université Pierre-et-Marie-Curie laboratoire de physiologie comparée (CNRS-U.A. 307). 4, place Jussieu, $75_{2} 30$ Paris Cedex 05 . 